EUGEN bietet dafür mehrere Optionen an:

- Dokumentenkamera: Es steht eine Videokamera zur Verfügung, mit der handschriftliche Notizen, Artikel- bzw. Buchseiten oder auch Lösungsskizzen direkt auf dem Teammonitor präsentiert und zur Diskussion gestellt werden können. Statt der Dokumentenkamera kann auch die Kamera des Smartphones verwendet werden, um das Bild mit der Teilen-Funktion des Smartphones auf dem Teammonitor darzustellen.

- Desktopsharing: Mit der Software VNC kann der gesamte Laptop-Bildschirminhalt direkt - als Livebild - auf dem Teammonitor dargestellt werden. Damit kann auf einfache Art und Weise interaktiv an einem Text gearbeitet werden. Für Smartphones und Tablets wird diese Funktionalität zur Zeit leider nicht angeboten.

- Dokumentenupload: Dokumente in üblichen Formaten (pdf, docx, odt, ...) lassen sich mittels der Verwaltungsoberfläche auf den Bildschirm hochladen und können abgebildet werden. Das funktioniert durch einfaches „Drag \& Drop“.

- Darstellung von Web-Seiten: Durch Eingabe einer URL wird auf dem Team-Bildschirm ein Browser gestartet und die Web-Seite geladen.

Es besteht die Möglichkeit, bis zu vier Dokumente gleichzeitig auf dem Monitor darzustellen. Zur angemessenen Präsentation gibt es mehrere Layout-Varianten. Somit können verschiedene Lösungsvarianten oder -ansätze parallel dargestellt, verglichen und besprochen werden.

Auch wenn zur Nutzung von EUGEN zunächst einige technische Hürden überwunden werden müssen, so steht unseren Benutzern mit EUGEN ein gutes Werkzeug zur Verfügung, um effektiv in Gruppen arbeiten zu können.

Stephan Abele

\title{
Info-Stelen in der WLB
}

\section{Aktuelle Informationen für unsere Benutzer}

Der Württembergischen Landesbibliothek ist es ein wichtiges Anliegen, unseren Benutzerinnen und Benutzern jeweils aktuelle Informationen und Wissenswertes über die Bibliothek mitzuteilen. Dazu werden unterschiedliche Kanäle bedient: Homepage, Soziale Medien, Newsletter, Infoblätter und Aushänge.

Seit einiger Zeit kommen nun auch Info-Stelen (Digital Signages) zum Einsatz.

Digitale Info-Stelen zeichnen sich durch ein großes Display, ein stabiles schlankes Gehäuse mit kippsicherem Fuß und einen „kleinen“ stromsparenden PC zur Auslieferung der Inhalte aus.

Neben der passenden Hardware wird jedoch auch entsprechende Software benötigt, um die Inhalte auf den verschiedenen Endgeräten verwalten zu können.

In der Einführungsphase wurden dazu die Inhalte in einer Powerpoint-Präsentation zusammenge-

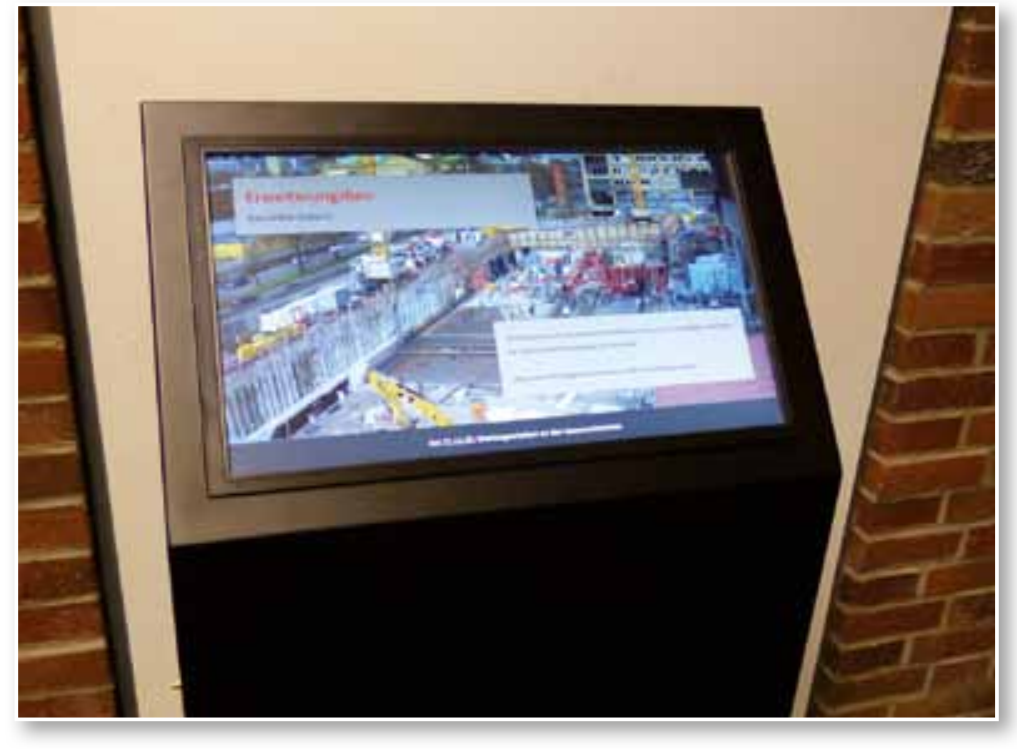

Abb. 1: Ausstellungsterminal

stellt und auf den Stelen in einer Endlosschleife abgespielt. Dies war jedoch kein optimaler Zustand, insbesondere, wenn mehrere Endgeräte zu bedienen waren. 


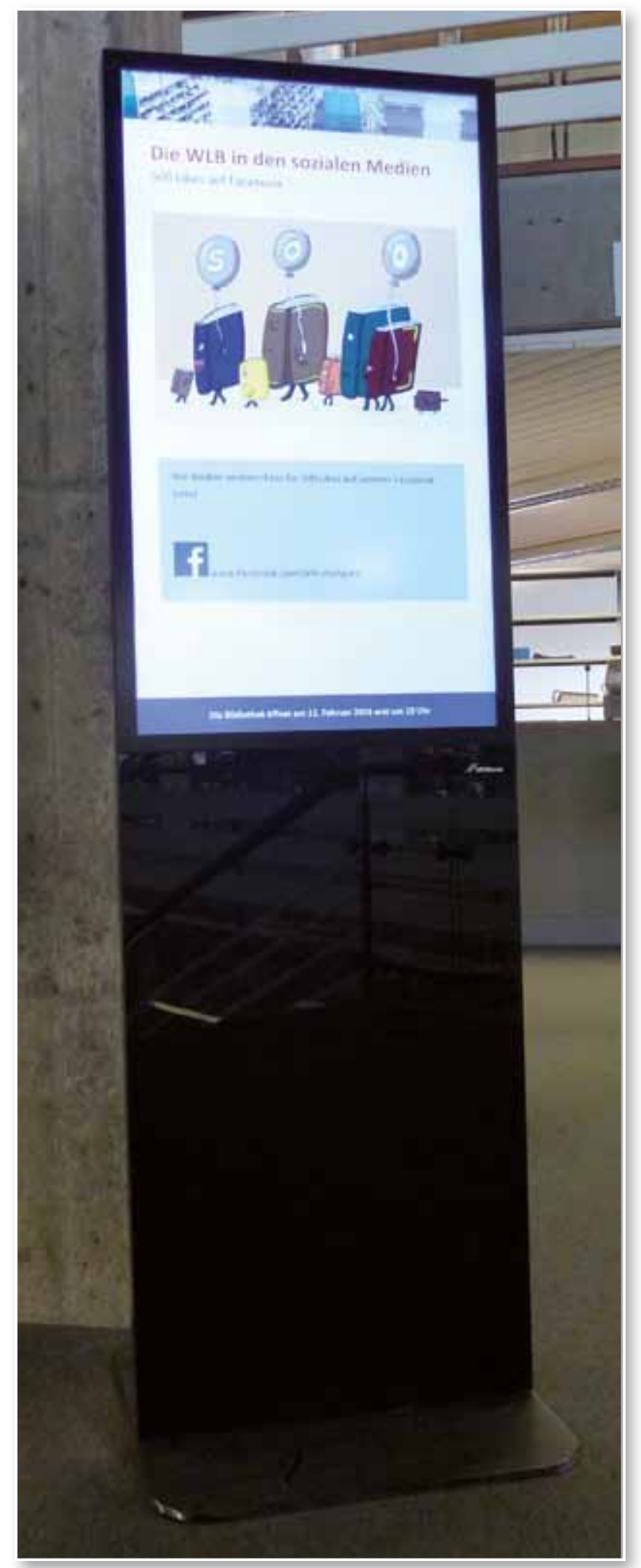

Abb. 2: Info-Stele

Zur Vorbereitung der Jubiläumsausstellung „250 Jahre Württembergische Landesbibliothek" wurde 2015 eine Spezifikation erarbeitet, die beschreibt, wie Ausstellungsinhalte und zeitliche Zusammenhänge (Zeitstrahl) präsentiert werden sollen.

Die Web-Agentur 3pc bietet bereits das Content Management System (CMS) e-publisher an.
Dieses CMS wurde gemäß der Vorgaben von 3pc um die Module "Zeitstrahl" und "Terminals" erweitert. Damit können webbasiert Inhalte und Medien verschiedenen Endgeräten zugeordnet und dort abgespielt werden. Auf den jeweiligen Ausstellungsgeräten selbst muss nur ein Browser verfügbar sein. Die Arbeit mit diesen Modulen hat sich sehr bewährt, so dass die Idee kam, diese Art und Weise der Bereitstellung von Inhalten auch für die Info-Stelen zu verwenden. „3pc" hat nach den Anforderungen der WLB im e-publisher ein weiteres Modul "Infostele" entwickelt.

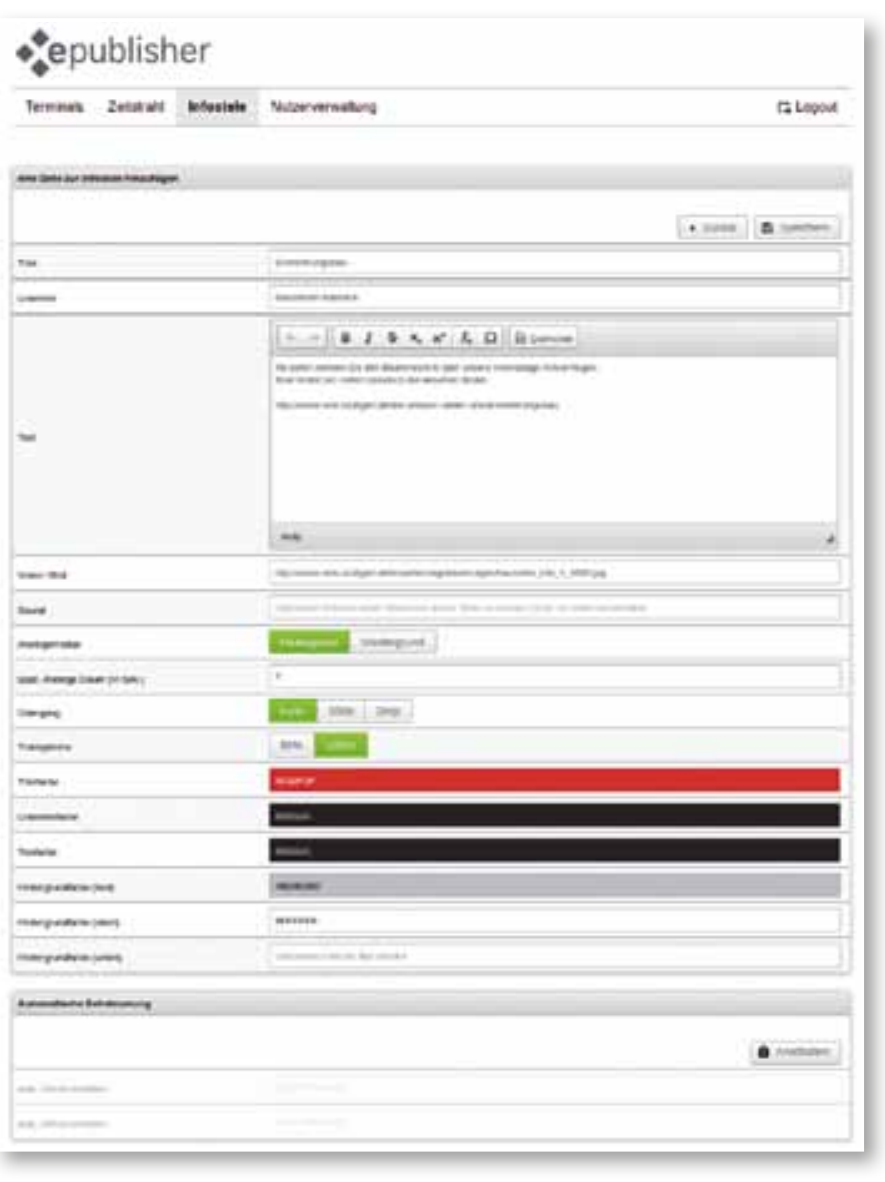

Abb. 3: Redaktionsansicht einer Seite (Quelle: http://3pc.de)

Mit diesem Werkzeug können im Browser beliebig viele unterschiedliche Info-Stelen verwaltet werden. Eine Info-Stele wird zunächst in der BackendAnsicht beschrieben und kann über eine URL von den Endgeräten aus abgerufen werden.

Die Beschreibung einer Info-Stele umfasst die Vorgabe einer Collage für die Kopfzeile, die Ausrichtung (Hochformat, Querformat) des Bildschirms und die Festlegung der Standard-Anzeigedauer der Seiten. Des Weiteren kann ein Lauftext für den unteren Rand festgelegt werden, der auf allen Seiten des jeweiligen Terminals dargestellt wird. Bei 
einem kurzen Text, der komplett auf eine Info-Stelenseite passt, kann die Animation auch abgeschaltet werden. Dies bewirkt eine angenehmere und ruhigere Darstellung. Die genannten Einstellungen bilden den Rahmen für eine Info-Stele, in den nun beliebig viele Seiten hinzugefügt werden können.

Wir haben uns - pro Bildschirmorientierung - für ein festes Layout entschieden, um die Arbeit für die "Redakteure" möglichst einfach und effizient zu halten. Es stehen die Inhaltselemente Titel, Untertitel, Bild bzw. Video und der beschreibende Text pro Seite zur Verfügung. Soll eine Seite eine abweichende Verweildauer erhalten, so kann diese hier ebenso festgelegt werden, wie Überblendeffekte und ggf. besondere Farbgestaltungen. Als Layoutvariante kann das Bild auch für den gesamten Hintergrund verwendet werden. Für manche Inhalte (z.B. Baustellen-Webcam) entstehen auf diese Weise interessantere Präsentationen. Über die Zeitsteuerung kann jeder Seite ein Sichtbarkeitszeitraum zugeordnet werden. Bei Termin gebundenen Inhalten entfällt dadurch der zeitnahe manuelle Eingriff und entsprechende Seiten können komfortabler vorbereitet werden.

In der Verwaltungsoberfläche steht eine Vorschaufunktion zur Verfügung, um Layout und Inhalte prüfen zu können. Da am Arbeitsplatz der Bearbeiter oftmals Bildschirme verwendet werden, deren Auflösung sich von der Info-Stelenhardware unterscheidet, ist dies ein sehr praktisches Feature.

In der Württembergischen Landesbibliothek besteht - u.a. auch durch die verschiedenen Baumaßnahmen - ein verstärkter Informationsbedarf unseren Nutzern gegenüber. Daher sollen im Bestandsgebäude in nächster Zeit noch weitere Info-Terminals aufgestellt werden.

Stephan Abele

\section{Kassenautomaten mit verbessertem Quittungsdruck}

Zur Bezahlung der verschiedenen Bibliotheksgebühren (Jahresgebühr, Portoersatz, Mahnungen usw.) stehen in der Württembergischen Landesbibliothek für unsere Benutzer bereits seit vielen Jahren zwei Kassenautomaten der Firma HESS zur Verfügung. Die Bezahlung ist sowohl mit Bargeld als auch mit der Girokarte möglich.

Die Kommunikation zwischen dem Bibliothekssystem aDIS/BMS und dem Kassenautomaten erfolgt mit dem NCIP-Protokoll. In der bisher eingesetzten Version wurde nur der jeweilige Saldo des Benutzerkontos zwischen Kassenautomat und Bibliothekssystem ausgetauscht. Der Nachteil dieser Lösung war, dass für den Benutzer kein detaillierter Quittungsdruck erstellt werden konnte. Wurde eine ausführliche Quittung benötigt, musste die Bezahlung an der Barkasse in der Leihstelle erfolgen. Im Zuge des aDIS/BMS-Update-Projektes wurde im Jahr 2015 die Erweiterung der NCIP-Schnittstelle insbesondere für Gebühreneinzelauflistung - durch die Firma aStec beauftragt. Auf der anderen Seite musste auch die Automatensoftware erweitert werden, so dass die umfangreicheren Daten, die aDIS ausliefert, verarbeitet werden können. Diese Software konnte im November 2015 fertiggestellt werden. Am 28.01.2016 wurde nun auf den Kassenautomaten diese neue Softwareversion installiert und auf aDIS/BMS-Seite die entsprechenden Erweiterungen aktiviert.

Der Benutzerdialog hat sich aufgrund der erweiterten Funktionalität etwas geändert.

Auf dem Bildschirm werden alle Gebührenposten dargestellt. Die primäre Sortierung erfolgt nach der Gebührenart (Nutzungsgebühr, Fernleihgebühr, usw.). Innerhalb der Gebührenart wird chronologisch sortiert. Zunächst sind alle Posten als „zu zahlen" aktiviert. Auf dem Touchbildschirm wird nun eine "Zahlen?"-Spalte angezeigt. Der Benutzer kann per Touchbedienung einzelne Posten von der Zahlung ausnehmen. Somit können beispielsweise 